\title{
96. A Characterization of the Second Order Elliptic Differential Operators
}

\author{
By Kôsaku YosidA \\ Department of Mathematics, University of Tokyo \\ (Comm. by Z. SuetunA, M.J.A., July 12, 1955)
}

§1. Introduction and the theorem. We consider the elliptic differential operator

$$
(E f)(x)=a^{i j}(x) \frac{\partial^{2} f}{\partial x^{i} \partial x^{j}}+b^{i}(x) \frac{\partial f}{\partial x^{i}}\left(a^{i j}(x) \xi_{i} \xi_{j} \geqq 0\right)
$$

as a linear operator from real-valued continuous functions $f(x)$ of the point $x=\left(x^{1}, \ldots, x^{m}\right)$ of an $m$-dimensional $C^{\infty}$ manifold $R$ to realvalued continuous functions $(E f)(x)$ of the point $x$. As was stressed by W. Feller, ${ }^{1)}$ it enjoys two important properties:

(1.2) The local property which means that the value $(E f)\left(x_{0}\right)$ is determined by the values of $f(x)$ in any small neighbourhood of $x_{0}$.

(1.3) The property $(E)$ which says that if $f(x)$ has a local minimum at $x_{0}$ then $(E f)\left(x_{0}\right) \geqq 0$.

W. Feller ${ }^{2)}$ has determined, for the case of one-dimensional $R$, the most general class of operators with these two characteristic properties. According to him such operator $E$ can, under the condition $E \cdot 1=0$, be represented by means of repeated differentiation $D_{v} D_{u} f$

with respect to monotone non-decreasing functions $u$ and $v$ (of these two functions, $u$ is a continuous function).

It is desirable to extend Feller's result to the case of higher dimensional $R$. The purpose of the present note is to give, by refining the method in a preceding note, ${ }^{3)}$ a partial answer to this problem. Our result gives a characterization of, so to speak, "the smallest closed extension" $A$ of the differential operator $E$.

Let us formulate the situation precisely. We assume that (i) $R$ is a homogeneous Riemann space, viz. $R$ is a $C^{\infty}$ Riemann space

1) W. Feller: The general diffusion operator and positivity preserving semi-groups in one dimension, Ann. Math., 60, No. 3, 417-436 (1954). W. Feller: On second order differential operators, Ann. Math., 61, No. 1, 90-105 (1955).

2) See the reference referred to in 1).

3) K. Yosida: On Brownian motion in a homogeneous Riemannian space, Pacific Journ. Math., 2, No. 2, 263-270 (1952). In this paper, the function space $C(R)$ can be corrected to be the Banach space which is the closure, by the norm defined by the maximum of the absolute value of the function, of the totality of continuous functions on $R$ with compact supports. 
such that the (Lie) group $G$ of isometries of $R$ is transitive on $R$, and we further assume that (ii) those isometries leaving a definite point $x_{0}$ invariant constitute a compact (Lie) subgroup of $G$. Let $A$ be a linear operator whose domain and range are real-valued functions on $R$. Following after W. Feller, we say that $f$ belongs to the domain of $A$ at $x_{0}$, in symbols, $f \in D\left(A: x_{0}\right)$, if both $f$ and $A f$ are defined and continuous in some neighbourhood of $x_{0}$. This domain $D\left(A: x_{0}\right)$ is supposed to be linear, that is, $f_{i} \in D\left(A: x_{0}\right)$ implies $\sum_{i} c_{i} f_{i} \in D\left(A: x_{0}\right)$. The operator $A$ is assumed to have (iii) the local property and (iv) the property $(E)$ for those $f$ which are contained in the intersection $D\left(A: x_{0}\right) \cap C^{\infty}\left(\left(x_{0}\right)\right)$. ${ }^{4)}$ Let, moreover, (v) $A \cdot 1=0$.

We shall say that (vi) $A$ is locally closed at $x_{0}$ if, whenever a sequence $\left\{f_{k}(x)\right\} \subseteq D\left(A: x_{0}\right)$ satisfies

(1.5) $\lim _{k \rightarrow \infty} f_{k}(x)=f_{\infty}(x), \lim _{k \rightarrow \infty}\left(A f_{k}\right)(x)=h_{\infty}(x)$ uniformly in a neighbourhood of $x_{0}$, then $f_{\infty} \in D\left(A: x_{0}\right)$ and $\left(A f_{\infty}\right)\left(x_{0}\right)=h_{\infty}\left(x_{0}\right)$.

We also say that (vii) $A$ is regular at $x_{0}$ if $D\left(A: x_{0}\right)$ includes functions arbitrarily and uniformly near to any continuous function which is $\neq 0$ at $x_{0}$ and 0 outside any small neighbourhood of $x_{0}$.

We may state our theorem as follows.

Theorem. Let the conditions (i)-(vii) be satisfied. Let us further assume that (viii) if $f \in D\left(A: x_{0}\right)$ then $f_{\alpha}(x)=f\left(T_{\alpha} x\right) \in D\left(A: x_{0}\right)$ for $T_{\alpha}$ in sufficiently small neighbourhood of the identity $T_{\alpha_{0}}$ of the group $G$ and $\left(A f_{\alpha}\right)(x)$ is continuous in the two variables $(x, \alpha)$ in some neighbourhood of $\left(x_{0}, \alpha_{0}\right)$. Then $D\left(A: x_{0}\right) \cap C^{\infty}\left(\left(x_{0}\right)\right)$ includes functions whose successive derivatives at $x_{0}$ are arbitrarily near to the corresponding derivatives at $x_{0}$ of any function $\in C^{\infty}\left(\left(x_{0}\right)\right)$, and, moreover, $(A f)\left(x_{0}\right)$ is, for $f \in D\left(A: x_{0}\right) \cap C^{\infty}\left(\left(x_{0}\right)\right)$, given by

$$
(A f)\left(x_{0}\right)=a^{i j}\left(x_{0}\right) \frac{\partial^{2} f}{\partial x_{0}^{i} \partial x_{0}^{j}}+b^{i}\left(x_{0}\right) \frac{\partial f}{\partial x_{0}^{i}} \text { with } a^{i j}\left(x_{0}\right) \xi_{i} \xi_{j} \geqq 0 .
$$

\section{$\S 2$. The proof of the theorem}

Lemma. Let $f \in D\left(A: x_{0}\right)$ and let a continuous function $g(x)$ vanish outside a sufficiently small neighbourhood of $x_{0}$. Then the "convolution"

$$
(f \otimes g)(x)=\int_{G} f\left(T_{\alpha} x\right) g\left(T_{\alpha} x_{0}\right) d \alpha
$$

belongs to $D\left(A: x_{0}\right)$. Here $d \alpha$ is a right invariant Haar measure of $G$.

Proof. The integral may be approximated by the Riemann sum

4) $C^{\infty}\left(\left(x_{0}\right)\right)$ denotes the totality of functions which are $C^{\infty}$ in some neighbourhood of $x_{0}$. Thus $D\left(A: x_{0}\right) \cap C^{\propto}\left(\left(x_{0}\right)\right)$ might be void except constant functions. However, see the theorem below. 


$$
\sum_{i} f\left(T_{\alpha_{i}} x\right) c_{i}
$$

uniformly in $x$ in a sufficiently small neighbourhood of $x_{0}$. This we see by the local uniform continuity of $f$, the condition (ii) and the fact that $g(x)$ vanishes outside a sufficiently small neighbourhood of $x_{0}$. By the condition (viii) we see that (2.2) and

$$
A\left(\sum_{i} f_{\alpha_{i}} c_{i}\right)(x)=\sum_{i}\left(A f_{\alpha_{i}}\right)(x) c_{i}
$$

both converges uniformly in a sufficiently small neighbourhood of $x_{0}$. Thus we see, by (vi), that (2.1) belongs to $D\left(A: x_{0}\right)$.

Corollary. There exist functions $f^{1}(x), \ldots, f^{m}(x) \in D\left(A: x_{0}\right) \cap C^{\propto}\left(\left(x_{0}\right)\right)$ such that the Jacobian

(2.3) $\partial\left(f^{1}(x), \ldots, f^{m}(x)\right) / \partial\left(x^{1}, \ldots, x^{m}\right)$ does not vanish at $x=x_{0}$.

There exists, for any system of $m^{2}$ constants $\alpha_{i j}$ with $\alpha_{i j}=\alpha_{j i}$, a function $f_{0}(x) \in D\left(A: x_{0}\right) \cap C^{\infty}\left(\left(x_{0}\right)\right)$ such that the values $\partial^{2} f_{0} / \partial x_{0}^{i} \partial x_{0}^{j}$ are arbitrarily near to the values $\alpha_{i j}$ respectively. In particular, there exists a function $f_{0}(x) \in D\left(A: x_{0}\right) \cap C^{\infty}\left(\left(x_{0}\right)\right)$ such that

$$
\left(x^{i}-x_{0}^{i}\right)\left(x^{j}-x_{0}^{j}\right)\left(\partial^{2} f_{0} / \partial x_{0}^{i} \partial x_{0}^{j}\right) \geqq 2 \sum_{i}\left(x^{i}-x_{0}^{i}\right) .
$$

Proof. It is possible, for $x$ in a sufficiently small neighbourhood of $x_{0}$, to choose $T(x) \in G$ such that

(2.5) $T(x) x=x_{0}$ and $T(x) x_{0}$ is a $C^{\infty}$ function of $x=\left(x^{1}, \ldots, x^{m}\right)$.

This we see from the fact that the set $\left\{T_{\alpha} \in G: T_{\alpha} x=x_{0}\right\}$ constitutes an analytic submanifold of $G$; it is one of the coset of $G$ with respect to the Lie subgroup $\left\{T_{\alpha} \in G: T_{\alpha} x_{0}=x_{0}\right\}$. Hence, by the right invariance of $d \alpha$, we have

$$
\begin{aligned}
(f \otimes g)(x) & =\int_{G} f\left(T_{\alpha} T(x) x\right) g\left(T_{\alpha} T(x) x_{0}\right) d \alpha \\
& =\int_{G} f\left(T_{\alpha} x_{0}\right) g\left(T_{\alpha} T(x) x_{0}\right) d \alpha .
\end{aligned}
$$

Thus, if $g$ is a $C^{\infty}$ function, we have

$$
\begin{aligned}
& \partial^{q_{1}+\cdots+q_{m}}(f \otimes g)(x) / \partial\left(x^{1}\right)^{q_{1}} \ldots \partial\left(x^{m}\right)^{q_{m}} \\
& =\int_{G} d \alpha f\left(T_{\alpha} x_{0}\right) \partial^{q_{1}+\cdots+q_{m}} g\left(T_{\alpha} T(x) x_{0}\right) / \partial\left(x^{1}\right)^{q_{1}} \ldots \partial\left(x^{m}\right)^{q_{m}} .
\end{aligned}
$$

Hence, by making use of the regularity of $A$ at $x_{0}$, we obtain the corollary. We have only to choose, in $(f \otimes g)(x) \in D\left(A: x_{0}\right), f$ and $g$ appropriately.

Proof of the theorem.5) We may take, by (2.3), the functions $f^{1}(x), \ldots, f^{m}(x)$ from $D\left(A: x_{0}\right)$ as the local coordinates of the point $x$ in a small neighbourhood of $x_{0}$. We shall denote these new local coordinates $f^{1}(x), \ldots, f^{m}(x)$ by the letters $x^{1}, \ldots, x^{m}$. Thus let

$$
\left(A x^{i}\right)\left(x_{0}\right)=b^{i}\left(x_{0}\right) \quad(i=1,2, \ldots, m) .
$$

The function $f_{0}(x)$ in the corollary belongs to $D\left(A: x_{0}\right)$. Hence, by the linearity of $D\left(A: x_{0}\right),(2.8)$, and the condition (v), we see that

5) Cf. the paper referred to in 3 ). 


$$
\begin{aligned}
\hat{f}_{0}(x) & =f_{0}(x)-f_{0}\left(x_{0}\right)-\left(x^{i}-x_{0}^{i}\right)\left(\partial f_{0} / \partial x_{0}^{i}\right) \\
& =\left(x^{i}-x_{0}^{i}\right)\left(x^{j}-x^{j}\right)\left(\partial^{2} f_{0} / \partial X^{i} \partial X^{j}\right)_{X=x_{0}+\theta\left(x-x_{0}\right)}
\end{aligned}
$$

belongs to $D\left(A: x_{0}\right)$.

Let us consider the subadditive ${ }^{6)}$ functional

(2.10) $\bar{A}(g)=\inf (A f)\left(x_{0}\right)$ for those $f \in D\left(A: x_{0}\right) \cap C^{\infty}\left(\left(x_{0}\right)\right)$ which satisfies the conditions: $f\left(x_{0}\right)=g\left(x_{0}\right)$ and $f(x) \geqq g(x)$ in a neighbourhood of $x_{0}$.

It is easy to see, by the property $(E)$ in (iv), that

$$
f \in D\left(A: x_{0}\right) \cap C^{\infty}\left(\left(x_{0}\right)\right) \text { implies }(A f)\left(x_{0}\right)=\bar{A}(f)=\underline{A}(f) \text { where }
$$

$$
\underline{A}(g)=-\bar{A}(-g) \text {. }
$$

We have, by (iii) -(iv) and (2.4),

$$
0 \leqq \bar{A}\left(\sum_{i}\left(x^{i}-x_{0}^{i}\right)^{2}\right) \leqq\left(A \hat{f}_{0}\right)\left(x_{0}\right)=\left(A f_{0}\right)\left(x_{0}\right)-b^{i}\left(x_{0}\right)\left(\partial f_{0} / \partial x_{0}^{i}\right)
$$

When $\partial^{2} f_{0} / \partial x_{0}^{i} \partial x_{0}^{j} \neq 0$, we have

$$
\begin{aligned}
& \left(\partial^{2} f_{0} / \partial X^{i} \partial X^{j}\right)_{X=x_{0}+\theta\left(x-x_{0}\right)}=\left(1+\varepsilon_{i j}(x)\right)\left(\partial^{2} f_{0} / \partial x_{0}^{i} \partial x_{0}^{j}\right) \\
& \text { where } \lim _{x \rightarrow x_{0}} \varepsilon_{i j}(x)=0 .
\end{aligned}
$$

Let $\varepsilon>0$. Then, by (2.13) and (iii)-(iv),

$$
\begin{aligned}
& A\left(\left(x^{i}-x_{0}^{i}\right)\left(x^{j}-x_{0}^{j}\right)\left(\partial^{2} f_{0} / \partial x_{0}^{i} \partial x_{0}^{j}\right)\right)-\varepsilon \bar{A}\left(\sum_{i}\left(x^{i}-x_{0}^{i}\right)^{2}\right) \\
& \leqq\left(A \hat{f_{0}}\right)\left(x_{0}\right) \\
& \leqq \bar{A}\left(\left(x^{i}-x_{0}^{i}\right)\left(x^{j}-x_{0}^{j}\right)\left(\partial^{2} f_{0} / \partial x_{0}^{i} \partial x_{0}^{j}\right)\right)+\varepsilon \bar{A}\left(\sum_{i}\left(x^{i}-x_{0}^{i}\right)^{2}\right) .
\end{aligned}
$$

Hence, by (2.12), we see that

$$
\left(A \hat{f}_{0}\right)\left(x_{0}\right)=\bar{A}\left(\left(x^{i}-x_{0}^{i}\right)\left(x^{i}-x_{0}^{j}\right)\left(\alpha^{2} f_{0} / \partial x_{0}^{i} \partial x_{0}^{j}\right)\right) .
$$

Similarly we obtain

$$
\left(A \hat{f}_{0}\right)\left(x_{0}\right)=\underline{A}\left(\left(x^{i}-x_{0}^{i}\right)\left(x^{j}-x_{0}^{j}\right)\left(\partial^{2} f_{0} / \partial x_{0}^{i} \partial x_{0}^{j}\right)\right) .
$$

We may choose, by the corollary, $f_{0} \in D\left(A: x_{0}\right)$ in such a way that $\partial^{2} f_{0} / \partial x_{0}^{i} \partial x_{0}^{j}$ are arbitrarily near to the given constants $\alpha_{i j}\left(=\alpha_{j i}\right)$ respectively. Thus we see, by the similar argument by which we have obtained (2.14) and (2.14)', that every quadratic form $\alpha_{i j}\left(x^{i}-x_{, j}^{i}\right)$ $\left(x^{j}-x_{0}^{j}\right)$ belongs to the domain of the linear functional $\tilde{A}$ defined by (2.15) $\widetilde{A}(g)=\widetilde{A}(g)=\underline{A}(g)$ if the latter two values are equal.

We have thus

$$
\widetilde{A}\left(\alpha_{i, j}\left(x^{i}-x_{0}^{i}\right)\left(x^{j}-x_{0}^{j}\right)\right)=\alpha_{i j} \tilde{A}\left(\left(x^{i}-x_{0}^{i}\right)\left(x^{j}-x_{j}^{j}\right)\right) .
$$

By repeating again the similar arguments, we see that any $f \in C^{\infty}\left(\left(x_{0}\right)\right)$ belongs to the domain of the linear functional $\tilde{A}$ and

$$
\begin{aligned}
& \tilde{A}(f)=\tilde{A}\left(f\left(x_{0}\right)+\left(x^{i}-x_{0}^{i}\right)\left(\partial f / \partial x_{0}^{i}\right)+\left(x^{j}-x_{0}^{j}\right)\left(x^{i}-x_{0}^{i}\right)\left(\partial^{2} f / \partial x_{0}^{i} \partial x_{0}^{i}\right)\right) \\
& =0+b^{i}\left(x_{0}\right)\left(\partial f / \partial x_{0}^{i}\right)+a^{j i}\left(x_{0}\right)\left(\partial^{2} f / \partial x_{i j}^{j} \partial x_{0}^{i}\right) \text {, where } \\
& a^{i j i}\left(x_{0}\right)=\tilde{A}\left(\left(x^{j}-x_{0}^{j}\right)\left(x^{i}-x_{0}^{i}\right)\right) \text {. }
\end{aligned}
$$

Hence, by (2.11), we have the theorem. That $a^{j i}\left(x_{0}\right) \xi_{j} \xi_{i}$ is $\geqq 0$ follows from the non-negativity of $\left(x^{j}-x_{j}^{j}\right)\left(x^{i}-x_{j}^{i}\right) \xi_{j} \xi_{i}$ and (iv).

6) $\bar{A}(g+h) \leqq \bar{A}(g)+\bar{A}(h)$ and $\bar{A}(\alpha g)=\alpha \bar{A}(g) \quad$ for $\alpha \geqq 0$. 